\title{
Sonderforschungsbereich SFB 738: Optimierung konventioneller und innovativer Transplantate
}

\author{
Autoren \\ Michael P. Manns, Petra Huber, Elmar Jaeckel \\ Institut \\ Medizinische Hochschule Hannover ( $\mathrm{MHH}$ ) und Helmholtz-Zentrum \\ für Infektionsforschung, Braunschweig (HZI)
}

\author{
Bibliografie \\ DOI http://dx.doi.org/10.1055/s-0043-101946 | \\ Z Gastroenterol 2017; 55: 1-4 @ Georg Thieme Verlag KG Stuttgart . \\ New York \\ ISSN 0044-2771
}

\section{Einleitung}

Der SFB 738 erforscht grundlegende Mechanismen, welche zum Transplantatversagen führen, sowie Mechanismen zur Rekurrenz von Grundkrankheiten nach Transplantation. Darüber hinaus sucht der SFB 738 nach Alternativen zur konventionellen Organtransplantation mit besonderem Schwergewicht auf Verfahren der Zellund Gentherapie, wie z. B. Hepatozytentransplantation. Seit ihrer Gründung gehört die Medizinische Hochschule Hannover $(\mathrm{MHH})$ zu den führenden Transplantationszentren, national wie international. An der $\mathrm{MHH}$ werden alle soliden Organtransplantationen durchgeführt (Leber, Niere, Lunge, Herz, Pankreas). Außerdem betreibt die $\mathrm{MHH}$ ein großes hämatopoetisches allogenes und autologes Stammzelltransplantationsprogramm sowohl für Erwachsene als auch für Kinder. Auch im Bereich der Lebendorganspende, vor allem der Niere, gehört die $\mathrm{MHH}$ zu den führenden Zentren. Während bei den soliden Organtransplantationen die Fortschritte der letzten Jahrzehnte vor allem auf der Entwicklung der chirurgischen Technik beruhten, ermöglicht die jahrzehntelange Erfahrung jetzt das Studium der Mechanismen zur chronischen Transplantatdysfunktion. Dabei gibt es für die verschiedenen Organsysteme gemeinsame, aber auch unterschiedliche, spezifische Probleme. Gemeinsam ist der generelle Anstieg des Alters der Organspender wie auch der Organempfänger. Spezifische Probleme entstanden in den letzten Jahren bei der Lebertransplantation durch den dramatischen Rückgang der Organspende und die Einführung des Meldesystems MELD zur Organallokation. Dadurch werden immer kränkere Patienten transplantiert; die Sterblichkeit wird von der Warteliste auf die Zeit nach der Transplantation verlagert. Bei der Niere besonders aktuell ist die Erforschung der chronischen Dysfunktion durch antikörpervermittelte Abstoßung (AMR), während bei der Lungentransplantation das Bronchiolitis-Obliterans-Syndrom (BOS) eine Hauptursache für die chronische Transplantatdysfunktion darstellt. Nach der
Lebertransplantation konnte die Rekurrenz von Grundkrankheiten, vor allem der Virushepatitiden, durch die therapeutischen Fortschritte der letzten Jahre verringert werden. In der Hämatologie ist die Rekurrenz der Leukämie nach Knochenmark- bzw. Stammzelltransplantation unverändert ein großes, bisher ungelöstes Problem. Die Rekonstitution des Immunsystems nach Transplantation mit rascher Wiederherstellung der Immunkompetenz gegenüber Infektionen stellt ein weiteres wichtiges und ungelöstes Problem dar, wobei gleichzeitig eine Graftversus-Host-Disease (GvHD) vermieden und eine Graftversus-Leukemia (GvL)-Reaktion erhalten bleiben soll. Die aktuellen Herausforderungen an die Transplantationsmedizin sind in $\mathbf{A b b} \mathbf{1}$ zusammengefasst.

\section{Projektbereiche}

Der SFB 738 gliedert sich in drei Projektbereiche plus den Bereich Z für zentrale Projekte ( $\checkmark$ Abb. 2 ). Im Projektbereich $A$ wird Immunität und Toleranz nach hämatopoetischer Stammzelltransplantation erforscht. Während Technologien der Stammzelltransplantation noch eine Zukunftsvision bei soliden Organtransplantationen darstellen, sind sie im Bereich der Hämatologie bereits praktizierter Alltag. Die Projekte im Bereich A beschäftigen sich u. a. mit Verbesserungen der Graft-versus-Leukemia-Reaktion (GvL) nach allogener, hämatopoetischer Stammzelltransplantation. Weitere Projekte erforschen die T-Zell-Regeneration nach hämatopoetischer Stammzelltransplantation und beschäftigen sich außerdem mit der Prävention der Graft-versus-Host-Disease (GvHD) durch zielgerichtete Inhibierung apoptoseassoziierter Proteine in aktivitierten Spender-T-Lymphozyten.

Im Projektbereich B werden die Determinanten für das Langzeitüberleben solider Organtransplantate studiert. Dabei stehen regulatorische T-Lymphozyten als therapeutischer Ansatz sowie die Verbesserung der Prävention der Hepatitis-Reinfektion nach Lebertransplantation im 


\section{Herausforderungen an die Transplantationsmedizin 2017}

\section{Solide Organtransplantation}

- Chronische Transplantatdysfunktion

- Mangel an Spenderorganen

- Immunologische Toleranz

- Humorale Abstoßung

- Rekurrenz der Grunderkrankung

\section{Stammzelltransplantation:}

- spezifische Graft vs. Leukemia Reaktion (GvL)

- Langsame Immunrekonstitution, Infektionen

- Graft vs. Host Disease (GvHD) Prophylaxe

\section{Alternativen zur klassischen Organtransplantation:}

- Zelltherapien (z.B. Hepatozyten, Pankreas-Inseln)

- Konditionierung von Transplantaten (z.B. ex vivo Perfusion)

- Genetische Modifikation von Transplantaten

- Genetische Korrektur angeborener Mutationen

- Abb. 1 Herausforderungen an die Transplantationsmedizin 2017.

Zentrum des Interesses. Weitere Projekte beschäftigen sich mit Mechanismen der Fibrogenese nach Organtransplantation mit besonderem Schwerpunkt auf chronischer Lungentransplantatdysfunktion. Durch ein besseres Verständnis Graft-spezifischer Toleranzmechanismen sollen die Voraussetzungen für einen therapeutischen Einsatz regulatorischer T-Lymphozyten geschaffen werden, um in Zukunft Langzeitorgantoleranz ohne toxische immunsuppressive Medikamente zu ermöglichen. Nach Herztransplantation ist die Post-Transplant-Arteriosklerose ein großes Problem, wahrscheinlich mitbedingt durch die bei dieser Organtransplantation besonders hochdosierte Immunsuppression. Am Tiermodell werden T-Zellvermittelte Immunreaktionen gegen Spendergewebe in vivo studiert, um dieses Problem besser zu verstehen und prophylaktische und therapeutische Ansätze zu entwickeln.

Der Projektbereich C beschäftigt sich u. a. mit der Entwicklung von Alternativen zur klassischen Organtransplantation, vor allem basierend auf molekularen und zellulären Therapieverfahren. Dabei werden grundlegende Mechanismen zur Etablierung stabiler alloantigenspezifischer Fox P 3-positiver regulatorischer T-Zellen (T-regs) erforscht. T-regs wiederum sollen zur Vermittlung aktiver spezifischer Immuntoleranz zum Einsatz kommen. Das zunehmende Alter sowohl der Organspender als auch der Transplantatempfänger stellt ein weiteres Problem dar. Deshalb wird am Beispiel der Nierentransplantation die Interferenz mit alterungsabhängigen Mechanismen als Strategie zur Transplantatverbesserung erforscht. Bei soliden Organen, wie z. B. der Leber, werden zur Korrektur einzelner Gendefekte seit Jahrzehnten orthotope Organtransplantationen durchgeführt. Dies bedeutet, dass ein komplettes Organ ausgetauscht wird, obwohl nur ein Gen defekt ist. Gentherapeutische Projekte sind daher besonders zukunftsorientiert. Unter Einsatz modernster Stammzelltechnologien, wie z. B. der CRISPR/CAS-Technologie, wird versucht, gezielte Genommodifikationen zu erreichen.

Die zentralen Projekte des SFB 738 nutzen u. a. die großen Technologieplattformen des Helmholtz-Zentrums für Infektionsforschung (HZI) in Braunschweig zur Identifikation, Isolation und molekularen Charakterisierung der verschiedenen Immunzellen. An der Medizinischen Hochschule Hannover gibt es außerdem ein Protokollbiopsieprogramm, welches Nieren-, Leber und Lungentransplantationen umfasst. Dieses wird auch vom SFB 738 unterstützt, da zahlreiche Projekte auf die im Rahmen des Protokollbiopsieprogramms gewonnenen Gewebe und andere Biomaterialien zugreifen müssen. 


\section{Projektbereiche und Projekte $\square$ SFB 738}

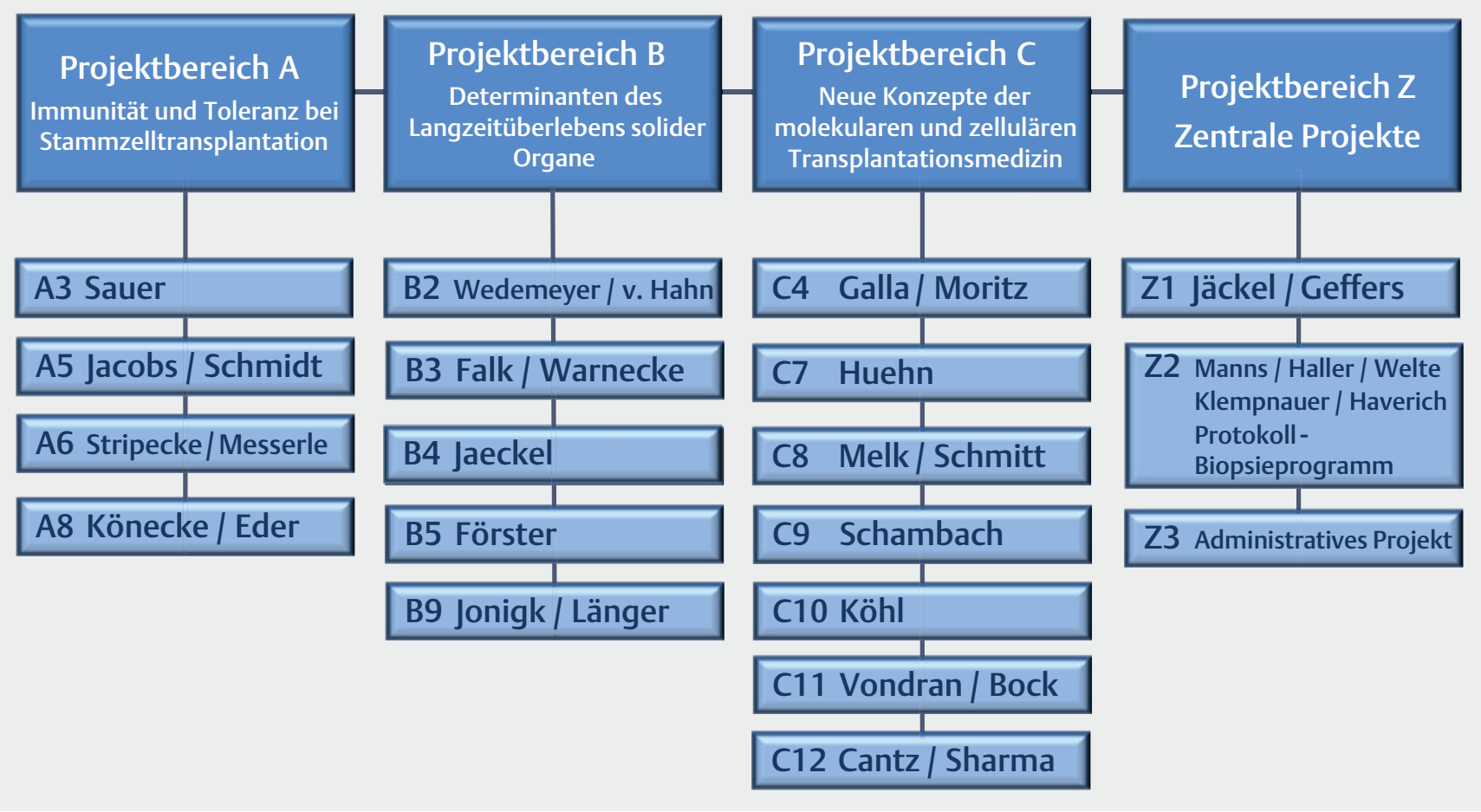

- Abb. 2 Die Struktur des SFB738.

\section{Einbindung des SFB 738 in lokale, na-} tionale und internationale Netzwerke

Die drei Forschungsschwerpunkte der MHH sind „Infektion und Immunität“, „Transplantation und Regeneration" sowie „Biomedizintechnik und Implantate“. Der SFB 738 ist integriert sowohl in den Forschungsschwerpunkt "Infektion und Immunität" wie auch in den Forschungsschwerpunkt „Transplantation und Regeneration“. Dabei kann der SFB 738 auf die bereits oben beschriebene jahrzehntelange Erfahrung im Bereich der klinischen Transplantationsmedizin der verschiedenen Organsysteme zurückgreifen. Vor allem für die zukunftsorientierten neuen Transplantationsverfahren wie der Stamm- und Zelltherapien sowie der Gentherapie kann sich der SFB738 auch auf die Erfahrungen der $\mathrm{MHH}$ im Bereich der hämatopoetischen Stammzelltransplantation, der Hepatozytentransplantation und der Therapie mit regulatorischen TLymphozyten berufen. Auch eine zentrale GMP-Einheit steht für die Herstellung zellulärer Transplantate zur Verfügung. Die Forschungsinfrastruktur der $\mathrm{MHH}$ im Bereich
Co-Sprecher: Arnold Ganser Koordination: Petra Huber 
ein gewisses nationales und internationales Alleinstellungsmerkmal, von einigen wird er sogar als Leuchtturmprojekt gesehen.

FAZIT

Der SFB 738 führt an der MHH alle wesentlichen Forschergruppen im Bereich der Transplantationsmedizin zusammen. Dies betrifft sowohl die verschiedenen soliden Organsysteme wie auch die hämatopoetische Stammzelltransplantation. Er stärkt wesentlich den Forschungsschwerpunkt „Transplantation und Regeneration" an der MHH. Sein Ziel ist es, durch Entwicklung einer gewebespezifischen Toleranz bei Erhalt zielgerichteter Immunität eine bessere Langzeitfunktion der verschiedenen Organtransplantate zu erreichen sowie durch innovative zelltherapeutische und gentherapeutische Verfahren Alternativen zur klassischen Organtransplantation zu entwickeln.

Website: www.sfb738.de

Unter www.sfb738.de ist auch ein DFG-unterstützter Imagefilm zu sehen.

\section{Danksagung}

Der SFB 738 und seine Mitglieder danken der Deutschen Forschungsgemeinschaft (DFG) für ihre jahrelange Unterstützung.

Interessenkonflikt

Die Autoren geben an, dass kein Interessenkonflikt besteht.

Korrespondenzadresse

Prof. Dr. med. Michael P. Manns, Sprecher SFB738 Petra Huber, Koordination SFB 738

Dr. med. Elmar Jaeckel, Wissenschaftlicher Sekretär SFB 738

Medizinische Hochschule Hannover (MHH)

Carl-Neuberg-Str. 1

30625 Hannover

Tel.: ++49/511/5326763

Fax: ++49/511/5325692

sfb738@mh-hannover.de 